\title{
BATTLESPACE TERRAIN OWNERSHIP: A NEW SITUATION AWARENESS TOOL
}

\author{
AUTHORS: \\ Janet F. O'May (POC) \\ Charles E. Hansen \\ Eric G. Heilman \\ Richard C. Kaste \\ Andrew M. Neiderer \\ U.S. Army Research Laboratory \\ ATTN: AMSRD-ARL-CI-CT \\ Aberdeen Proving Ground, MD 21005-5067 \\ 410-278-4998 (voice) \\ 410-278-4988 (fax) \\ jomay@arl.army.mil, \\ charlie@arl.army.mil, \\ heilman@arl.army.mil, \\ rck@arl.army.mil, \\ neiderer@arl.army.mil
}




\title{
BATTLESPACE TERRAIN OWNERSHIP: A NEW SITUATION AWARENESS TOOL
}

\begin{abstract}
Information in the battlespace provides decisive power. It is imperative that critical information is brought to the forefront to enhance decision-making. An accurate model of a tactical operation will improve a commander's battlespace awareness. One vital piece of information is terrain control. The Battlespace Terrain Ownership (BTO) system embodies an algorithm that computes expected terrain control over time and space, based on combat power projection as a function of position, influence exerted by asset distribution, weapon system effectiveness, probabilities of hit and kill, and combat damage.
\end{abstract}

\section{Introduction}

Situation Awareness (SA) has been defined as "the perception of the elements in the environment within a volume of time and space, the comprehension of their meaning and the projection of their status in the near future."[1] A good military SA tool integrates disparate information to facilitate battlespace understanding. Reducing voluminous tactical information provided by sensors, combat platforms, and soldier reports into a less complex form enables a commander to perceive the repercussions of military actions in both operation planning and battle execution.

An understanding of the forces required to control critical terrain features can cue planners to the numbers and types of combat units optimal for an operation. Further, a commander can gauge how well a battle is progressing by knowing which of his forces control significant terrain. Future commanders should have the ability to assess control at any time in a quick and efficient manner. We use computation and graphic display to present dynamic battlespace terrain control information in a form immediately useful and intuitively graspable. Our team is developing a prototype system that provides a graphical visualization of combat asset effectiveness in gaining and maintaining control of battlespace regions.

\section{Background}

The U.S. Army Research Laboratory (ARL) mission includes providing combat force readiness through innovative technology. Battlespace Decision Support Team (BDST) research focuses on developing techniques that meet the fundamental challenge of ensuring battlefield dominance via software tools designed for mobile commanders. Our novel treatment of combat modeling facilitates the evaluation of combat planning effectiveness and thereby improves force readiness.

BDST research is part of two ongoing ARL programs. First, BDST has explored the applicability of combat simulation to networks, information, and integration. Specifically, our endeavors have centered on course of action (COA) evaluation and metrics for the planning/replanning process of modern combat. Our objectives include the development and evaluation of COA decision methodologies for mobile battlefield commanders, the establishment of connections between constructive simulations and actual battle, the extension of combat modeling mathematics to $\mathrm{COA}$ analysis, and the creation of technology evaluation programs for fundamental research products within Army facilities. In particular, we are applying our data mining techniques to simulation results to analyze tactical operations.

Second, in FY04 we prototyped an algorithm and program, jointly known as Battlespace Terrain Ownership (BTO), that visually represents opposing forces' control of a battlespace. The algorithm is incorporated into software that graphically displays a color-coded representation of both "owned" and neutral conflict areas. Dynamically changing a terrain control diagram as a battle progresses should aid the commander in timely prediction of crucial tactical events. Our approach using a visual representation of terrain "ownership" lends itself to both combat simulation and eventually actual battlespace monitoring systems. We have linked the prototype into streaming data from a One Semi-Automated Forces (OneSAF) execution to demonstrate dynamic updating of an ownership display based on real-time battlefield information. In FY05 we are solidifying this work as a dynamic decision aid prototype and extending it into the urban operations domain with a graphical user interface.

\section{Applicability to Current and Future Forces}

\subsection{Mission Planning}

The importance of BTO lies in the fact that it can be used in different stages of the battle. With its current hookup to a combat simulation, BTO can assist in the mission planning phase. BTO can provide possible physical 
ranges of advance, e.g., a scenario can be configured in a combat simulation and executed numerous times to determine possible entity locations during and after the mission. The types and quantities of ammunition can be evaluated to augment the basic load of the entities. The fit of equipment on hand and the mission can be analyzed to determine if changing equipment or altering a mission would result in an improved battle outcome. By utilizing multiple iterations of a simulation BTO may help identify mission-critical features and assist in establishing objectives.

\subsection{Battle Execution}

BTO currently executes based on simulation feeds, however future work will incorporate real-world data from disparate sources. As BTO receives information from sensors and intelligence sources, it can serve as a monitor of battle execution. This will provide many advantages to include: a quick reference to each force's control of the battlespace; a color coded battle map that delineates the active battle area and projects the power exerted by each force on the battlespace; an evaluation of the advancement of the forces in the time allotted for tasks; and an understanding of "success" or the degree of mission completion.

\subsection{After Action Review}

An after action review is critical to evaluating the success of tactics or missions. As BTO creates the displays of terrain control it saves the data. This data can be used to review the battle execution. A completed battle can be reviewed for battle performance. This will provide a "lessons learned" capability for the commander and staff. An additional advantage is the discovery of new parameters through the mining historical data. New battle parameters will increase the understanding of battlespace interactions.

\section{System Configuration}

The BTO system is designed to reduce critical battlespace information into an easily understood format using a visual display. During development, we used a laboratory test environment to produce combat data utilized by the BTO algorithm. BTO computes terrain control dynamically based on combat power projection as a function of position, influence exerted by asset distribution, weapon system effectiveness, probabilities of hit and kill, and combat damage. Using this information BTO creates a graphical display of the battlespace featuring zones of combat power to yield an understanding of terrain control.
We have recently made several improvements to the BTO program under the auspices of the Command and Control in Complex and Urban Terrain Army Technology Objective. We improved the OneSAF-Ownership interface so that it requires reduced human interaction. This effort facilitates initiation of the linkage between the combat simulation and the decision aid, for which the prior system required a knowledgeable operator due to differences in networked hardware. Ongoing efforts include development of a Java-based graphical user interface (GUI) for launching the ownership tool, porting the tool to a Linux environment, and improved plotting routines (based on research into formats for Java3D/browser). These efforts will improve portability of the program, leading to feedback from soldiers at the Army's Battle Command Battle Laboratory on decision aid prototyping.

\subsection{Battlefield Data}

The design of BTO calls for input sources containing actual battlefield data. To emulate these sources in laboratory conditions, we used a combat simulation, OneSAF, during prototype development. We modified the OneSAF simulation program to produce data files containing both force interactions (combat) and entity status (logistics).[2] Further, the team developed a Southwest Asia scenario for experimentation in combat metrics. Battle data generated through the execution of the team's scenario underpins BTO development.

The Southwest Asia scenario depicts a company-sized assault on a numerically superior prepared threat defense. The terrain is typical of that found in the Southwest Asia theatre of operations. As is shown in Figure 1, the attacking force consists of thirteen M1 main battle tanks.[3] The threat defense represents a reduced strength battalion.

Figure 2 is a rough drawing of the scenario. The friendly force is split into two groups, Eastern and Western, each attacking along a route converging on an objective designed to control transportation in the area. Both the Eastern and Western forces must contend with a river crossing and navigating a railroad. The river is indicated by the thick blue line and the railroad by the heavy dotted gray line. The Eastern forces initially secure the flank of the main effort force (Western approach) by attacking the town. (The town is indicated by the six-sided hashed figure.) Once taken, this force proceeds to the company objective of the railroad. The Western attack first seizes the railroad bridges and, once the town to the East is secure, moves to take the railroad objective. 


\author{
Attacking Forces (By Attack Route): \\ One Company (-) \\ East Attacker: 5 M1 Main Battle Tanks \\ West Attacker: 8 M1 Main Battle Tanks \\ Defending Forces (By Defending Battle Position): \\ One Mixed Battalion (-) \\ WEST \\ Band 1: 2 T-80 Main Battle Tanks \\ 3 BMP-2 Infantry Fighting Vehicles \\ Band 2: 2 T-72M Main Battle Tanks \\ 3 T-72M Main Battle Tanks \\ 2 T-72M Main Battle Tanks \\ Band 3: 2 T-72M Main Battle Tanks \\ Band 4: 2 T-80 Main Battle Tanks

\section{EAST} \\ Band 1: 3 BMP-2 Infantry Fighting Vehicles \\ 2 BMP-2 Infantry Fighting Vehicles \\ Band 2: 3 T-72M Main Battle Tanks \\ Band 3: 1 T-80 Main Battle Tank \\ Band 4: 1 T-80 Main Battle Tank
}

Figure 1. Scenario Force Composition

The defense is laid out in a "band" configuration. (The bands are designated by the heavy black arcs in Figure 2.) Each band contains a mix of vehicles designed to weaken and finally defeat an attacking unit. The bands are static for this scenario.

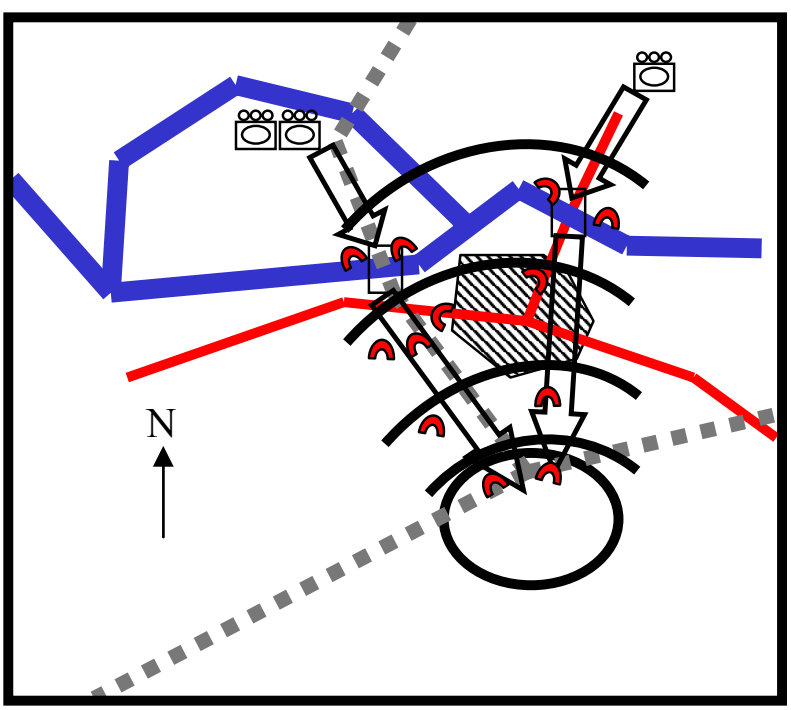

Figure 2. Scenario Schematic Diagram
While the data produced includes only direct fire results, a significant understanding of terrain control emerges through a calculation of combat weapons' effects. The BTO examples that follow illustrate the ratio of combat power present at points during execution of this scenario. Using BTO, commanders will quickly understand and respond to the developing combat situation.

\subsection{Data Preprocessing}

A critical part of BTO is to take simulation data from OneSAF, pass this to the control algorithms, and then graphically display the resultant calculations. To perform this link, we developed a Java 2 Standard Edition Version 5.0 (J2SE 5.0) application program.[4] The "write once, run anywhere" philosophy of Java provides the necessary platform portability for BTO.

The main method of our application gathers position and status information for all entities in a battle area during a OneSAF scenario run.[5] An executing scenario results in direct fire, logistic, and vehicle data. New records are automatically detected when they are produced by the simulation. The detection of new simulated output triggers an update and is used to produce the next display of a BTO frame.

\subsection{BTO Algorithm}

As a battle command decision aid, BTO will display areas of the battlefield controlled by each force with the degree of control classified into one of six categories. Commanders will be able to ascertain the battlefield situation at a glance. To compute terrain control, we assume that combat system capabilities are known for all weapons in both friendly (Blue) and enemy (Red) forces, and that Blue and Red vehicle status and position are noted as they change. The BTO algorithm partitions the battlefield into a number of rectangular areas for individual examination. Given a distribution of vehicles on the battlefield and subsequent positions as the battle progresses, we seek to determine the collective power that the combatant forces are able to project onto each individual rectangular area. The expected power projected, or more accurately the expected benefit, will be our measure of control or ownership.

We assume that for a given vehicle, any vehicle type of the opposing force may occupy a partitioned area we are considering. For our scenario if we look at a Blue vehicle, this means that a T72, T80, or BMP2 may occupy the partitioned area being analyzed; in the case of a Red vehicle, an M1 tank may be located there. The vehicle types would change by the scenario configuration. We also assume that each vehicle may fire whatever rounds are available to that weapon system. From data collected 
in a number of OneSAF runs we have the probability of a certain round hitting a certain target. We also have available the probability of the various kill types ( $\mathrm{M}$ for mobility, $\mathrm{F}$ for firepower, MF for mobility and firepower, and $\mathrm{K}$ for catastrophic) given a hit for each type round against each type vehicle. This probability is a function of range, round dispersion, angle of attack, and hit location. Finally, we have a subjective estimate of the benefit accruing to the shooter for each kill type where a hit resulting in no damage has a benefit of 0 and a hit resulting in a $\mathrm{K}$ kill has a benefit of 1 . Other kill types have benefit values between 0 and 1 and can be set by the user. For a single vehicle, the average maximum power projected onto an area is the product of the probability of hit, the probability of the various kill types given a hit, and the benefit accruing for each kill type where we vary the round, target, location of the hit, and the angle of attack.

This maximum power will be degraded to reflect the overall distribution of combatants in the battlespace. The approach used is to consider both friendly and enemy vehicles within range of the vehicle of interest $(\mathrm{V})$ and to calculate a probable effect that the distribution of all vehicles may have on V's ability to project the maximum power available to it. The degraded power projected will be termed usable power. The usable power of $\mathrm{V}$ is calculated by degrading maximum power based on the number of active enemy vehicles within range of $\mathrm{V}$ and the power projected by the friendly vehicles onto the enemy vehicles threatening $\mathrm{V}$. The collective usable power of Blue and Red is calculated and then the percentage of total usable power is determined for Blue and Red. The ratio of these percentages is the measure of our degree of ownership for that individual area. For example, if Blue owns $25 \%$ of one area and Red owns the remaining $75 \%$, then the ratio of Blue ownership is $1 / 3$, while the ratio of Red ownership is 3. The process is repeated for each partitioned area.

For plotting purposes, we show the ownership for the force having the advantage. We use six classifications: the ratio is $>=3: 1$ (Blue and Red), the ratio is $>=2: 1$ and $<3: 1$ (Blue and Red), the ratio is $>=1: 1$ and $<2: 1$ (Blue and Red combined), and an un-owned class where, because of weapon range restrictions, neither force has any control over an area. Areas for each class are color coded and plotted. We also color code the individual vehicles and differentiate between undamaged vehicles and damaged vehicles. We can also plot, as a function of time, the number of active vehicles and an overall percentage of ownership in the active area.

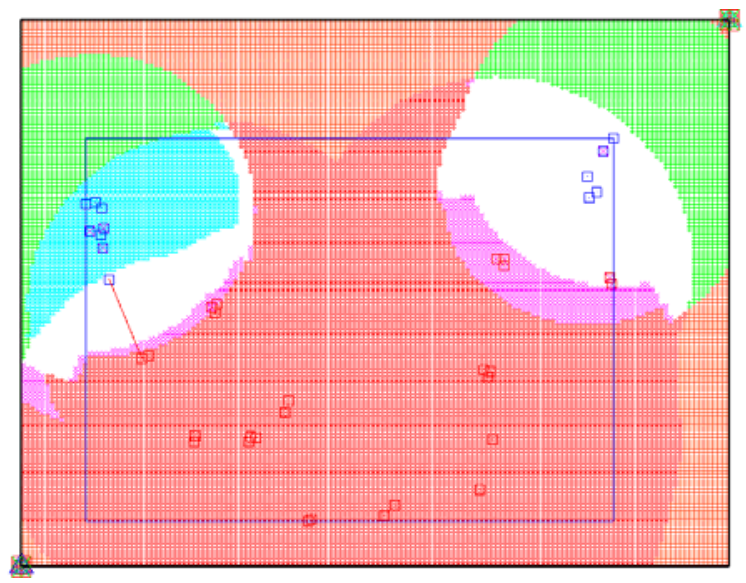

Plot 1

The color coded ownership plot is updated each time there is a direct fire hit or a change in vehicle position occurs. Plot 1, as an example, shows the ownership situation at some point during a battle. Blue vehicles appear as blue boxes, Red vehicles as red boxes with an empty interior or an $\mathrm{X}$ to indicate an undamaged or damaged vehicle respectively. Blue/Red areas where ownership is $>=3: 1$ are shown in green/red respectively, $>=2: 1$ and $<3: 1$ in blue/purple, $>=1: 1$ and $<2: 1$ are white, and un-owned areas in a lighter shade of red. The active area rectangle is shown in blue. The numeric values of the percentage ownership for each rectangular area within the active area are summed for both Blue and Red and an average percentage ownership is calculated. These are plotted and shown in Plot 2. Plot 3 shows the number of active (undamaged) vehicles during the course of this battle.

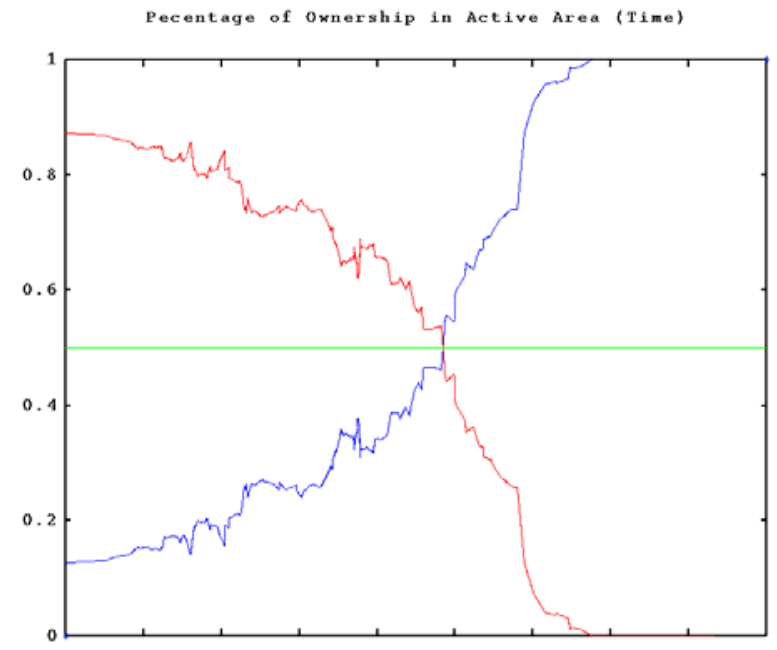

Plot 2 
The BTO prototype does not currently take into account a LOS calculation to determine whether combatants can see each other. Investigations into LOS have been going on for decades. For example, [6] describes attributes needed for determining LOS and the capabilities of various algorithms, in particular the interpolation post method used in OneSAF. Differences between such algorithms are based both on the methods used to determine elevations at points not coincident with post elevations and methods used to determine location points to be analyzed. Our research requires non-probabilistic techniques that can be generalized into an urban environment. We are developing an algorithm specifically geared to our application. Unlike other algorithms of which we are aware, this does the calculation for a function that handles all the points along the LOS.

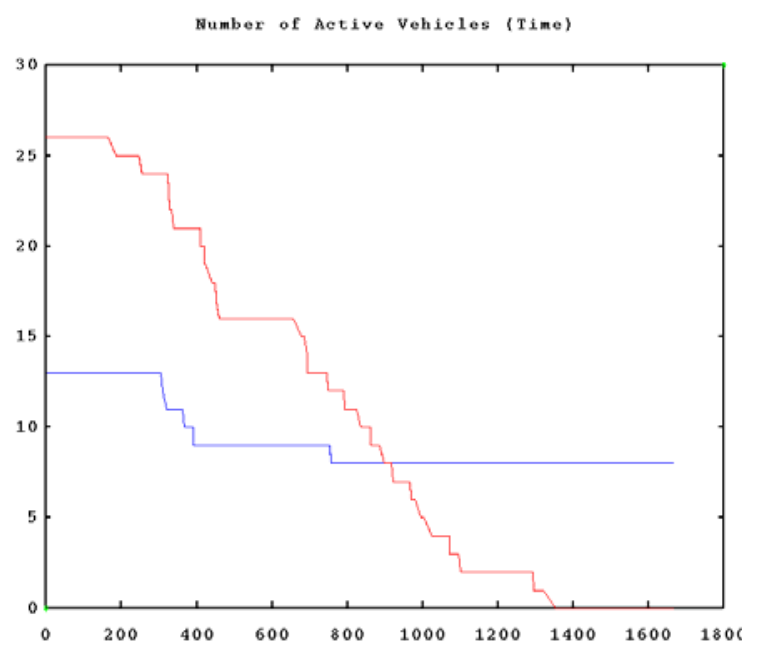

Plot 3

We assume, reasonably, that terrain z-elevations are available on an xy-grid. We determine a quadratic polynomial, using bilinear interpolation, representing the terrain elevation of all $(\mathrm{x}, \mathrm{y})$ along the projection of the LOS onto the xy-plane in the grid square as a function of a variable. This variable represents the difference between the $\mathrm{x}$-coordinate of the point and the $\mathrm{x}$-coordinate of the origin of that grid square divided by the width of the grid square in the $\mathrm{x}$-direction.

We can also determine a linear expression for the elevation along the projection of the LOS in the xy-plane as a function of the same variable hx as was used above. If we set these two expressions equal to each other and solve the resulting quadratic equation, a solution lying in the grid square indicates an obstructed LOS in that grid square.

\section{Future Work}

The BTO system will continue to gain capabilities. One of our goals is to provide a BTO tool that is user-friendly without compromising any existing capability. Our next release of BTO will include a Java GUI for launching each individual application and OneSAF.

Currently, the BTO uses a legacy graphics package for terrain display. The package, called gnuplot, is written in the FORTRAN computer language and provides a limited number of features for the display of terrain control. The adoption of J2SE 5.0 as a prototype integration language led to the consideration of newer, more capable graphics packages written to be compatible with Java. We are examining the multi-purpose graphics package called JPlot written in Java with improved graphics options.[7] To also improve the visualization, future work will include registering the generated terrain control plot on top of a two-dimensional map. We plan to integrate BTO with other current and developmental mapping products.

\section{Conclusion}

The benefits of our modeling and analysis capability are two-fold. First, the approach allows an information pull by the commander and staff of a small, select subset of key battlefield parameters, lending insight to unfolding battle events. The mathematical analysis of a combat simulation, due to the high resolution of the data, will uncover the effects of individual parameters or interactions that would ordinarily not be obvious. Time saved in fundamental battle analysis will enable a commander's staff to comprehensively evaluate several COAs in the time normally used for a single COA. Second, information expressed in terms of a conditional probability of success given specific battle status parameters during execution will assist in the determination of contingency plan initiation. BTO may influence the set of critical information requirements tracked by the commander during execution.

BTO is the first in a statistically-based automated tool set capable of producing battlespace analyses that describe occurrences key to the success of a military operation. BTO renders a dynamic real-time display of terrain ownership designed to improve battle SA. While the prototype receives data from simulated sources, the final version will monitor actual battlefield sensor feeds. Our team will sponsor a series of experiments to quantify the worth of this technology. Tools such as BTO will enable rapid understanding of the situation.

Novel techniques for military decision aiding will facilitate the creation of important tools for the combat 
simulation and analysis communities and also enable an understanding of battlespace metrics' impact on planning and execution. Our analyses will establish decision points for advising real-world commanders on the options of continuing or replanning an ongoing battle. Decision aids such as BTO will assist the commander in maintaining battlefield initiative. BTO enables a real-time visualization of possible weakness on the battlefield and is presented without overloading the commander with too many details.

This work has the potential for improving military battle command and analogous civilian systems. Other beneficiaries could include any activity requiring rapid evaluation of operational plan status: in particular emergency responders. Our project could assist commanders to recognize force potentials and incorporate terrain control in battle monitoring and execution.

\section{References}

[1] M. Endsley: "Design and evaluation for situation awareness enhancement" in Proceedings of the Human Factors Society 32 ${ }^{\text {nd }}$ Annual Meeting (pp.97-101), 1988.

[2] For more information on the modifications to OneSAF and the team's combat metric experimentation, see: Heilman, Eric G., Janet F., O’May, One SemiAutomated Forces (OneSAF) Killer/Victim Scoreboard (KVS) Capability, ARL-TR-2829, Aberdeen Proving Ground, MD, 2002.

[3] O'May, Janet, Eric Heilman, Barry Bodt, Joan Forester, Identifying Battlefield Metrics Through Experimentation, Proceedings of the $7^{\text {th }}$ ICCRTS, Quebec City, Quebec, 2002, p.5.

[4] Additional information on Java can be found at http://java.sun.com/j2se/1.5.0/index.jsp.

[5] The format of OneSAF output can be reviewed at http:/www.onesaf.org. Any specific questions concerning the actual translation, which was done in Java, is available upon request and should be directed to the Battlespace Decision Support Team at ARL.

[6] M. Proctor and W. Gerber, "Line-of-Sight Attributes for a Generalized Application Program Interface," Journal of Defense Modeling and Simulation, , Vol. 1, Issue 1, April 2004, pp. 43-57.

[7] For more information on the JPLOT program, see: http://www.cig.ensmp.fr/ vanderlee/jplot/. 